\title{
Evaluación del potencial de lodos orgánicos carbonizados de una planta de tratamiento de aguas residuales para el mejoramiento de suelos
}

\author{
Sánchez, Graciela \\ Facultad de Ingeniería Mecánica, Universidad Tecnológica de Panamá \\ Veraguas, Panamá \\ graciela.sanchez@utp.ac.pa \\ Ramírez, Joisleen \\ Facultad de Ingeniería Mecánica, Universidad Tecnológica de Panamá \\ Los Santos, Panamá \\ joisleen.ramirez@utp.ac.pa \\ James, Arthur \\ Facultad de Ingeniería Mecánica, Universidad Tecnológica de Panamá \\ Panamá, Panamá \\ Sistema Nacional de Investigación (SNI) \\ Secretaría Nacional de Ciencia Tecnología e Innovación (SENACYT) \\ arthur.james@utp.ac.pa \\ Deago, Euclides \\ Facultad de Ingeniería Civil, Universidad Tecnológica de Panamá \\ Panamá, Panamá \\ euclides.deago@utp.ac.pa \\ Villarreal, José \\ Laboratorio de suelos, Instituto de Investigación Agropecuaria de Panamá \\ Herrera, Panamá \\ jevilla38@gmail.com
}

\begin{abstract}
In Panama, organic sludge from wastewater treatment plants (WWTP) is not fully used. The carbonization of these organic sludge is presented as an alternative for the management of this organic waste. The carbonization of biomass produces a rich-carbon material known as biochar. Besides the carbon content, this material contains nutrients that could improve the physicochemical properties of the soil and provide porosity that would enhance water and
\end{abstract}


nutrient retention. In Panama, $27 \%$ of the soils are degraded due to irregular fertilizer and pesticide application practices. The most critical areas of soil degradation are Cerro Punta, the Comarca Ngöbe Buglé, the Sabana Central Veragüense and the Arco Seco. The objective of this project was to evaluate biochar production using mixtures of rice husk and biosolids from a WWTP. Mixtures of $85 \%, 75 \%, 65 \%$, and $55 \%$ organic sludge were used, and the remaining percentage represented the amount of rice husk for each of the corresponding mixtures. The results showed the need to add volatile matter to the mixture for the proper operation of the reactor. The mixture of $75 \%$ organic sludge and $25 \%$ rice husk produced biochar with the most potential to improve the physicochemical properties of the soil. It presented a pH of 12.25 and productivity of $35.48 \%$. The use of organic matter from charred waste is projected as an alternative for improving degraded soils and promoting a circular economy and sustainable development.

Keywords: Biochar, organic sludge, WWTP, soils, rice husk.

\section{Resumen}

En Panamá, los lodos orgánicos de las plantas de tratamiento de aguas residuales (PTAR) no se utilizan en su totalidad. La carbonización de estos lodos se presenta como una alternativa para la gestión de este residuo orgánico. La carbonización de la biomasa produce un material rico en carbono conocido como biocarbón. Además del contenido de carbono, este material contiene nutrientes que podrían mejorar las propiedades fisicoquímicas del suelo y proporcionar porosidad que mejoraría la retención de agua y nutrientes. En Panamá, el $27 \%$ de los suelos están degradados debido a prácticas irregulares de aplicación de fertilizantes y pesticidas. Las áreas más críticas de degradación del suelo son Cerro Punta, Comarca Ngöbe Buglé, la Sabana Central Veragüense y el Arco Seco. El objetivo de este proyecto fue evaluar la producción de biocarbón utilizando mezclas de cáscara de arroz y biosólidos de una PTAR. Se utilizaron mezclas de $85 \%$, 75\%, 65\% y 55\% de lodos orgánicos, y el porcentaje restante representó la cantidad de cascarilla de arroz para cada mezcla. Los resultados mostraron la necesidad de agregar materia volátil a la mezcla para el correcto funcionamiento del reactor. La mezcla de $75 \%$ de lodo orgánico y $25 \%$ de cáscara de arroz produjo biocarbón con el mayor potencial para mejorar las propiedades fisicoquímicas del suelo. Presentó un pH de 12,25 y una productividad de 35,48\%. El uso de materia orgánica procedente de residuos carbonizados se proyecta como una alternativa para mejorar los suelos degradados y promover una economía circular y un desarrollo sostenible.

Palabras claves: Biocarbón, lodos orgánicos, PTAR, suelos, cascarilla de arroz. 


\section{INTRODUCCIÓN}

La biomasa residual es un tipo de biomasa que se genera como residuo a partir de diferentes actividades. Estos residuos pueden ser biomasa de origen animal y vegetal, residuos agrícolas y lodos orgánicos [1]. Estos últimos, son desechos sólidos de origen biológico procedentes de las plantas de tratamiento de aguas residuales (PTAR). Los mismos están compuestos de materia orgánica y nutrientes [2]. La energía de los lodos orgánicos se puede aprovechar utilizando tratamientos térmicos, como pirólisis y termoquímicos, como la gasificación. Durante el proceso de gasificación se pueden obtener productos como syngas, bio-oil y biocarbón, en presencia de un agente oxidante y a levadas temperaturas [3], [1].

El biocarbón, se define como "un sólido obtenido de la conversión termoquímica de biomasa en un ambiente con poco oxígeno" [4]. Este producto es capaz de mejorar las propiedades fisicoquímicas del suelo. Por lo que, se considera como una enmienda para los suelos, ya que podría incrementar la productividad de los cultivos, aporta fertilidad al suelo a largo plazo, incrementa sus nutrientes. Además, este podría corregir los efectos negativos por acidez o alcalinidad [5], [6].

El biocarbón a partir de lodos orgánicos podría aumentar la disponibilidad de nutrientes y restaurar los suelos forestales deteriorados [7]. Este presenta la capacidad de mejorar la estructura, filtración, respiración y retención de líquidos en el suelo [8]. Un estudio comparó el biocarbón de lodos orgánicos con el biocarbón de estiércol de vaca y el compost de lodos orgánicos, en donde se identificó que este posee un mayor contenido de nitrógeno, óxido de calcio y óxido de fósforo [6]. Por otra parte, un estudio identificó que el biocarbón de lodos orgánicos puede reducir la lixiviación de metales pesados en el suelo, entre ellos $\mathrm{Cu}$, $\mathrm{Ni}$ y Zn comparado con el lodo orgánico sin carbonizar [6]. La carbonización de los lodos orgánicos permite la inmovilización de los metales pesados y agentes tóxicos [9].

El uso excesivo de agroquímicos ha formado parte de las malas prácticas de producción agropecuaria que han conducido al aumento del deterioro y degradación de los suelos y con ello la inseguridad alimentaria [10]. A largo plazo estos inhiben la capacidad del suelo a retener nutrientes y agua, provocando que la fertilidad y la materia orgánica disminuyan. La degradación del suelo contribuye al cambio climático mediante las emisiones de gases efecto invernadero y reduce las tasas de absorción de carbono [11].

En Panamá, el 27\% del territorio presenta suelos en estado de degradación. Las áreas más afectadas se ubican en Cerro Punta, Comarca Ngöbe Buglé, Sabana Central Veragüense y el Arco Seco [10]. El aprovechamiento de la biomasa residual permite disminuir la acumulación de residuos, debido a revalorización de la materia prima [1], [3]. En Panamá, aproximadamente, se vierten 70 Ton/día de lodos orgánicos al relleno sanitario Cerro 
Patacón, proveniente de la PTAR de la Ciudad de Panamá [10], [11]. Debido a esta situación, en este proyecto se evalúa el potencial de los lodos orgánicos carbonizados como una alternativa para el mejoramiento de suelos.

\section{MÉTODO}

\section{A. Muestreo y pretratamiento de la materia prima}

El lodo orgánico (L.O) se obtuvo de la PTAR de la Ciudad de Panamá ubicada en el corregimiento de Juan Díaz. El lodo orgánicos es estabilizado mediante óxido de calcio (CaO) o cal ligera en una dosificación 136,08 g de cal/g de lodo orgánico [12]. Además, este debe cumplir con la norma DGNTI-COPANIT 472000 de Panamá [13], la cual indica los parámetros apropiados para su disposición y aplicación como abono o producto agrícola. Los lodos orgánicos estabilizados recolectados en la PTAR fueron secados por 15 días a temperatura ambiente. Posteriormente, fueron secados en un horno (Royal Range of California INC., California, EE. UU) a una temperatura de $250^{\circ} \mathrm{C}$ por 2 horas. Adicionalmente, se agregó cascarilla de arroz al lodo orgánico, debido al bajo contenido de materia volátil que este contiene. En Panamá se genera aproximadamente 1534610 Ton/año de cascarilla de arroz, lo cual representa a la cosecha de $93200 \mathrm{Ha}$ sembradas. Este residuo agroindustrial se obtuvo del Molino Doferra S.A., ubicado en Panamá, corregimiento de 24 de diciembre. Se realizaron diferentes mezclas de lodos orgánicos y cascarilla de arroz, en las siguientes proporciones. Los lodos orgánicos se agregaron en proporciones en peso de $85 \%$ (B85), 75\% (B75), 65\% (B65) y 55\% (B55) y el porcentaje restante representó la cantidad de cascarilla de arroz para cada una de las mezclas correspondientes. Estos son porcentaje en peso de cada materia prima. Estas mezclas fueron tamizadas en un rango de tamaño entre 1,18 y 4 $\mathrm{mm}$.

\section{B. Carbonización de biomasa}

La producción de biocarbón se realizó en un gasificador de flujo ascendente tipo top lit (TLUD). Se suministró aire con un compresor de 1,5HP - 150 psi de presión de operación máxima y de 6 galones de reserva (BRIGGSS \& STRATTON, Wisconsin, EE. UU.). El flujo de aire se evaluó a $20 \mathrm{~L} / \mathrm{min}$ y se controló con un medidor de flujo de área montado en aluminio ((RITEFLOW ${ }^{\circledR}$ FLOWMETERS de BEL-ART, Wayne, NJ, EE. UU), escala $150 \mathrm{~mm}$, tamaño $\mathrm{N}^{\circ} 4$. Se utilizaron 2 termopares tipo K (diámetro de 0,318 cm) en la parte superior e inferior del gasificador. Las temperaturas se registraron con un sistema de adquisición de datos (Onset, modelo UX120, Bourne, MA, EE. UU) y para la obtención de estas, se utilizó el software $\mathrm{HOBO}^{\circledR}$ (versión 3.7.19).

La biomasa se distribuyó a lo largo del reactor hasta cubrir la parte superior de este. El periodo de ignición fue de 1 minuto y se utilizó gas propano para facilitar el encendido. Los 
gases de combustión salían a través de una chimenea ajustada al gasificador TLUD. Además, se utilizó láminas de corcho como aislante entre las superficies de la chimenea y el reactor.

\section{Técnicas de análisis}

La metodología utilizada para analizar las muestras fue similar a la realizada por Bethancourt et al., 2009 [14]. Se realizó análisis químico para evaluar carbono orgánico (C.O), nitrógeno $(\mathrm{N})$, óxido de fósforo $\left(\mathrm{P}_{2} \mathrm{O}_{5}\right)$, óxido de potasio $\left(\mathrm{K}_{2} \mathrm{O}\right)$, óxido de calcio $(\mathrm{CaO})$, óxido de magnesio $(\mathrm{MgO})$, manganeso $(\mathrm{Mn})$, hierro (Fe), cobre ( $\mathrm{Cu}$ ) y zinc $(\mathrm{Zn})$. Estos parámetros fueron determinados por un espectrofotómetro de absorción atómica de fuente continua, marca Analytik Jena (AA300, Alemania). La materia orgánica de la muestra se analizó a través de la metodología de digestión húmeda de Walkley-Black. Además, se evaluó el pH de las muestras. Estos análisis se realizaron en el Laboratorio de Fertilidad de Suelo del Instituto de Investigación Agropecuaria de Panamá (IDIAP).

\section{RESULTADOS}

En la tabla 1, se presenta la composición química del biocarbón obtenido. La temperatura en las muestras de biocarbón presentaron un aumento progresivo en las mezclas de B75, B65, B55 con una correlación de R2 =0,78. Esto indicó que, a menor cantidad de lodo orgánico en la mezcla, la temperatura de carbonización aumentó. Mientras, que el rendimiento disminuyó a medida que la temperatura aumentó en las mezclas B75, B65 Y B55. No obstante, la productividad fue mayor en B85, lo que coincide con el estudio de Huang et al., 2017. En donde demuestran que a mayor temperatura la productividad del biocarbón disminuye [15].

Por otra parte, el pH aumentó con respecto al mayor porcentaje de lodo orgánico en la mezcla, ya que presentó una correlación de $\mathrm{R}^{2}=0,45$. El nivel del $\mathrm{pH}$ interviene con la disponibilidad de nutrientes en el biocarbón y en el suelo. Un $\mathrm{pH}$ ácido en el suelo contribuye a la presencia de $\mathrm{Al}, \mathrm{Cu}, \mathrm{Fe}, \mathrm{Mn}$ y $\mathrm{Zn}$. Mientras que, un $\mathrm{pH}$ básico favorece el contenido de $\mathrm{P}, \mathrm{Ca}, \mathrm{Mg}$, y $\mathrm{K}$ [16]. Los nutrientes en el biocarbón pueden disminuir en el proceso de volatilización durante la carbonización, debido a las altas temperaturas. La temperatura de volatilización para el $\mathrm{C}$ es de $100^{\circ} \mathrm{C}$, para el $\mathrm{N}$ aproximadamente $200^{\circ} \mathrm{C}$. Mientras que, $\mathrm{P}$ y K pueden volatilizarse a $800^{\circ} \mathrm{C}$ y los nutrientes como $\mathrm{Mn}, \mathrm{Mg}$ y Ca se volatilizan a temperaturas mayores de $1000^{\circ} \mathrm{C}$ [17]. Lo cual conlleva a una disminución de $\mathrm{N}$, carbono orgánico (C.O), materia orgánica (M.O).

Estudios demuestran que, al aumentar las temperaturas de $300^{\circ} \mathrm{C}$ a $700^{\circ} \mathrm{C}$ el biocarbón obtenidoa partir de lodos orgánicos presenta una disminución de N, M.O y de la productividad. No obstante, K, P, Zn, Mn, Mg, Cu, Ca, Fe contenidos en el lodo orgánico aumentaron con la carbonización [18]. El carbono orgánico (C.O) disminuyó durante el proceso de carbonización, debido al aumento de la temperatura. Sin embargo, no presenta una correlación con la 
temperatura de reacción. Esto no coincide con los resultados registrado por Huang [15]. El análisis de varianza de Tukey demostró que los resultados son significativamente diferentes. Por otra parte, la M.O disminuyó en el proceso de carbonización, ya que el aumento de temperatura, en un rango de $300^{\circ} \mathrm{C}$ a $900{ }^{\circ} \mathrm{C}$, descompone la materia orgánica [15].

Tabla 1. Composición química del biocarbón obtenido a diferentes mezclas de biomasa.

\begin{tabular}{|c|c|c|c|c|c|c|c|c|c|c|c|c|c|c|}
\hline \multirow[t]{2}{*}{ Muestra } & $\mathrm{pH}$ & $\begin{array}{c}\text { Tempe- } \\
\text { ratura }\end{array}$ & $\begin{array}{l}\text { Produc- } \\
\text { tividad }\end{array}$ & C.O & M.O & $\mathrm{N}$ & P2O5 & $\mathrm{K} 2 \mathrm{O}$ & $\mathrm{CaO}$ & $\mathrm{gO}$ & $\mathrm{Mn}$ & $\mathrm{Fe}$ & $\mathrm{Zn}$ & $\mathrm{Cu}$ \\
\hline & & ${ }^{\circ} \mathrm{C}$ & \multicolumn{8}{|c|}{$\%$} & \multicolumn{4}{|c|}{$\mathrm{mg} / \mathrm{l}$} \\
\hline L.O & 10,8 & & & 3,15 & 5,43 & 4,90 & 3,06 & 0,25 & 16,79 & 1,68 & 38 & 1840 & 550 & 110 \\
\hline Casca & 5,8 & & & 4,60 & 7,94 & 0,64 & 0,050 & 0,44 & 0,24 & 0,06 & 147 & 170 & 78 & 15 \\
\hline B55 & 12,33 & 1254,31 & 29,11 & 0,19 & 0,33 & 0,2 & 6,53 & 0,43 & 26,05 & 6,84 & 216,67 & 5907 & 4410 & 290 \\
\hline B65 & 12,67 & 1130,72 & 31,77 & 0,72 & 0,9 & 0,22 & 6,76 & 0,43 & 25,43 & 5,45 & 217,33 & 4654 & 3919 & 264,33 \\
\hline B75 & 12,33 & 1080,78 & 35,48 & 0,01 & 0,03 & 0,21 & 6,94 & 0,39 & 25,63 & 6,27 & 213,33 & 6107 & 2095,33 & 255,67 \\
\hline B85 & 13 & 1087,31 & 37,94 & 0,05 & 0,09 & 0,13 & 6,67 & 18,5 & 32,54 & 6,40 & 202,67 & 1392 & 840,67 & 255,33 \\
\hline
\end{tabular}

(85\% lodos orgánicos y 15\% cascarilla de arroz (B85), 75\% lodos orgánicos y 25 \% cascarilla de arroz (B75), 65\% lodos orgánicos y 35 \% cascarilla de arroz (B65) y 55\% lodos orgánicos y 45 \% cascarilla de arroz

El nitrógeno disminuyó a medida que la concentración de lodo orgánico aumentó con una correlación de $R^{2}=0,48$. Los porcentajes de este se mantienen entre $0,13 \%$ y $0,22 \%$, los cuales no son significativamente diferentes según el análisis de varianza de Tukey. La proporción de $\mathrm{P}_{2} \mathrm{O}_{5}, \mathrm{~K}_{2} \mathrm{O}, \mathrm{CaO}$ aumentaron con respecto a la disminución de la temperatura [18]. Además, tiende a disminuir a medida que la concentración de cascarilla de arroz es mayor. La cantidad de $\mathrm{MgO}$ aumentó en el proceso de carbonización, siendo $1.68 \mathrm{mg} / \mathrm{L}$ para el lodo orgánico (L.O) y $6,84 \mathrm{mg} / \mathrm{L}$ para B55, que posee la temperatura más alta de reacción $\left(1254,31^{\circ} \mathrm{C}\right)$.

La proporción de Mn aumentó en los biocarbones 216,67, 217,33, 213,33, 202,67 mg/L para B55, B65, B75, B85, respectivamente, en comparación con L.O (38 mg/L). Se infiere que se debe al proceso de carbonización y al agregado de la cascarilla de arroz, ya que este nutriente está presente en un $147 \mathrm{mg} / \mathrm{l}$. Por otra parte, el Fe es otro nutriente que está disponible en el lodo orgánico (L.O, $1840 \mathrm{mg} / \mathrm{L}$ ) y en la cascarilla de arroz (Casca, $170 \mathrm{mg} / \mathrm{L}$ ). Además, se observa que este elemento aumentó en el proceso de carbonización, siendo la proporción más alta en el biocarbón B55 con $25907 \mathrm{mg} / \mathrm{L}$. En la literatura científica, se ha demostrado que este micronutriente esencial, suministrado en pequeñas dosis al suelo no es perjudicial [19].

El micronutriente Zn, se comportó de forma similar al Fe, ya que aumentó su disponibilidad en las muestras de biocarbón, en comparación con el lodo orgánico (L.O). La muestra B85 presentó 5840,67 mg/L, mientras que B55 registró $4410 \mathrm{mg} / \mathrm{L}$ en su composición. Sin 
embargo, no existe una correlación entre las variables, por lo que se realizó análisis de varianza de Tukey. Lo cual demostró que las variables son significativamente diferentes. Este nutriente es un fertilizante natural, por lo que beneficia al suelo [20]. Mientras que, el Cu presentó un aumento en su disponibilidad con el proceso termoquímico. La mayor proporción de este elemento se presenta en la mezcla B55 (290 mg/L), seguido por B65, B75 y $B 85(264,33,255,67,255,33 \mathrm{mg} / \mathrm{L}$, respectivamente). Además, se deduce que el aumento de Cu se debe a la disminución de la cantidad de lodos orgánicos en la mezcla carbonizada.

\section{CONCLUSIONES}

La adición de cascarilla de arroz al lodo orgánico permitió la carbonización de la biomasa debido al mayor contenido de materia volátil que posee la cascarilla de arroz. El biocarbón obtenido mostró resultados distintos en cuanto a temperatura, siendo el biocarbón B55 el que obtuvo la mayor temperatura de reacción y la menor productividad. No obstante, el biocarbón B85 obtuvo la mayor productividad. El biocarbón B75 presentó características estables en cuanto a los niveles óptimos de los nutrientes requeridos por los suelos en la Región de Azuero. La carbonización de esta biomasa en un gasificador TLUD podría contribuir a la mejora de los suelos, ya que es una alternativa sostenible con valor añadido y a la vez, podría promover una economía circular.

\section{Referencias}

[1] J. A. Carta, R. C. Pérez, A. Colmenar, and M. Castro, Centrales de energías renovables. Generación eléctrica con energías renovables. 2007.

[2] E. González Flores, M. A. Tornero Campante, Y. Ángeles Cruz, and N. Bonilla Y Fernández, "Concentración total y especiación de metales pesados en biosólidos de origen urbano," Revista Internacional de Contaminacion Ambiental, vol. 25, no. 1, pp. 15-22, 2009.

[3] H. Yang and H. Chen, "Biomass gasification for synthetic liquid fuel production," Gasification for Synthetic Fuel Production. Woodhead Publishing, pp. 241-275, Jan-2015.

[4] IBI, "Standardized Product Definition and Product Testing Guidelines for Biochar That Is Used in Soil," International Biochar Initiative, p. 23, 2015.

[5] J. Lee, A. K. Sarmah, and E. E. Kwon, "Production and Formation of Biochar," Biochar from Biomass and Waste, pp. 3-18, 2019.

[6] [J. E. Amonette and S. Joseph, Biochar for Environmental Management. 2009.

[7] G. Gascó, B. Gutiérrez, and A. Méndez, "Soil biochemical activities and the geometric mean of enzyme activities after application of sewage sludge and sewage sludge biochar to soil," pp. 511517, 2012.

[8] A. Méndez, A. Gómez, J. Paz-ferreiro, and G. Gascó, "Chemosphere Effects of sewage sludge biochar on plant metal availability after application to a Mediterranean soil," vol. 89, pp. 1354-1359, 2012.

[9] P. Devi and A. K. Saroha, "Risk analysis of pyrolyzed biochar made from paper mill effluent treatment plant sludge for bioavailability and eco-toxicity of heavy metals," Bioresource Technology, vol. 162, pp. 308-315, Jun. 2014. 
[10] M. de Ambiente, Estrategia Nacional de Cambio Climático, 2050 Panamá., vol. 166, no. 3611. 2019.

[11] L. Olsson et al., "Land Degredation," Climate Change and Land: an IPCC special report on climate change, desertification, land degradation, sustainable land management, food security, and greenhouse gas fluxes in terrestrial ecosystems, pp. 345-436, 2019.

[12] Ministerio de ambiente, Atlas De Las Tierras Secas y Degradadas De Panamá. 2009.

[13] Ministerio de Comercio e Industrias, "Reglamento técnico DGNTI-COPANIT 47-2000.Usos y disposición final de lodos," Gaceta Oficial de la República de Panamá, 2000.

[14] G. Bethancourt, A. James, J. E. Villarreal, and N. Marin-Calvo, "Biomass carbonization - production and characterization of biochar from rice husks," Proceedings - 2019 7th International Engineering, Sciences and Technology Conference, IESTEC 2019, pp. 40-45, 2019.

[15] H. jun Huang, T. Yang, F. ying Lai, and G. qiang Wu, "Co-pyrolysis of sewage sludge and sawdust/rice straw for the production of biochar," Journal of Analytical and Applied Pyrolysis, vol. 125, pp. 61-68, 2017.

[16] J. E. Villarreal, I. Ramos, J. Villalaz, and A. Santo, “Clasificación taxonómica y caracterización físicoquímica de los suelos de la región de Azuero-Panamá," pp. 1-7, 2017.

[17] T. H. DeLuca, M. D. MacKenzie, M. J. Gundale, and D. . Jones, "Biochar effects on soil nutrient transformations," Biochar for Environmental Management: Science and Technology, vol. 2, pp. 421-454, 2015.

[18] H. Yuan, T. Lu, H. Huang, and D. Zhao, "Journal of Analytical and Applied Pyrolysis Influence of pyrolysis temperature on physical and chemical properties of biochar made from sewage sludge," Journal of Analytical and Applied Pyrolysis, vol. 112, pp. 284-289, 2015.

[19] E. C. Luque, "Propiedades magnéticas de óxidos de hierro en suelos mediterráneos," Universidad de Córdoba, 2008.

[20] L. Dyner et al., "Composición y aporte potencial de hierro, calcio y zinc de panes y fideos elaborados con harinas de trigo y amaranto," Latinoamericanos de Nutrición, vol. 57, no. 1, pp. 69-78, 2007.

\section{Autorización y Licencia CC}

Los autores autorizan a APANAC XVIII a publicar el artículo en las actas de la conferencia en Acceso Abierto (Open Access) en diversos formatos digitales (PDF, HTML, EPUB) e integrarlos en diversas plataformas online como repositorios y bases de datos bajo la licencia Attribution-NonCommercialShareAlike 4.0 International (CC BY-NC-SA 4.0) https://creativecommons.org/licenses/by-nc-sa/4.0/. Ni APANAC XVIII ni los editores son responsables ni del contenido ni de las implicaciones de lo expresado en el artículo. 